\title{
Ethernet-Based Passive Optical Local-Area Networks for Fiber-to-the-Desk Application
}

\author{
Igor Radovanović, Student Member, IEEE, Wim van Etten, Senior Member, IEEE, and Hennie Freriks
}

\begin{abstract}
We introduce optical local-area network (LAN) architectures based on multimode optical fiber and components, short wavelength lasers and detectors, and the widely used fast Ethernet protocol. These architectures are designed to lower the costs associated with passive optical LAN implementation. Further reduction in overall cost is achieved through decreased network downtime, lower maintenance cost, extended geometrical spans, and larger headroom for future capacity increase. These optically transparent networks represent a novel approach for implementing fiber-to-the-desk.
\end{abstract}

Index Terms-Collision detection, embedded clock transport, fast Ethernet, fiber-to-the-desk, passive optical local-area networks.

\section{INTRODUCTION}

$\mathbf{T}$ HE extensive growth of Internet traffic at the end of the last century contributed to telecom industry boom, leading to massive deployment of optical fiber primarily in core and metropolitan networks. Although optical local-area network (LAN) standards are available [1], [2], copper interconnects continue to be more dominant. Gigabit Ethernet technology is in wide use thanks to Category $5 \mathrm{e}$, although connection distances have been reduced and technical problems more prevalent. Finding a new solution for the fiber-optic network that will be used in fiber-to-the-desk (FTTD) application, which will bring down the overall implementation cost while offering better performance than copper-based network, remains a challenge.

A problem that designers encounter is that sometimes networks have to operate in environments that are "polluted" by interfering electromagnetic radiation, e.g., power plants, airport buildings, military facilities, etc. In these environments, unshielded twisted pair (UTP) transmission can be subject to long packet delay and inherently low throughput. Optical fibers, on the other hand, offer an excellent nonemissive transmission medium and at the same time are completely insensitive to incoming radiation.

Increased interest in using optical fiber all the way to individual PCs is manifest in larger companies where storage area networking, data backup demands, and sharing access to large databases [3] are of great importance. These firms are willing to invest in optical fiber when upgrading their networks since they offer larger headroom for future bandwidth increase. Nevertheless, for as long as the overall cost of fiber network remains high, they will continue to use the copper medium. Furthermore,

Manuscript received December 2, 2002; revised July 1, 2003.

The authors are with Telecommunication Engineering Group, University of Twente, 7500AE Enschede, The Netherlands (e-mail: i.radovanovic@ieee.org). Digital Object Identifier 10.1109/JLT.2003.819145 to exploit fiber running to the desktop in smaller offices and homes, the overall cost of the network must be even lower. An additional impediment is the need for optoelectrical conversion in the network interface card (NIC). In the future these costs may be overcome by using low-cost CMOS technology with the receiver front-end monolithically integrated with the photodetector [4], [5].

In this paper, two broadband passive optical LAN architectures are introduced. The proposed networks have short downtime, low maintenance cost, extended geometrical spans compared with the network span specified in the short-wavelength optical LAN standard [2], and larger headroom for future capacity increase. These networks are novel because it is the first time that the fast Ethernet protocol has run on a passive optical star network. As a consequence, a few changes at the physical layer level had to be implemented, since the newly designed networks use a common medium. These changes have to do with the collision detection method and the synchronization of bursty traffic, which are the main issues that are discussed in this paper.

Presented optical LANs are based on the most widely used standard, IEEE $802.3 \mu$, namely, 100BASE-T. Most contemporary computers use this standard. The fiber-optic data transport standard 100BASE-FX cannot be used since we propose point-to-multipoint transmission in contrast to the point-to-point transmission in 100BASE-FX networks.

To achieve affordable application of optical transmission in the LANs, we have used multimode fibers, which are less vulnerable for misalignment and dust particles and short wavelength components to make the system more economically attractive. Laser diodes (LDs) and vertical-cavity surface-emitting laser (VCSELs) operating at $850 \mathrm{~nm}$ are less expensive than lasers operating at 1300 and $1550 \mathrm{~nm}$. They also open the possibility of integrating a $\mathrm{Si}$ photodiode with the receiver front-end using straightforward CMOS technology. The LDs that we have used in the network realization show relaxation oscillation at a frequency well beyond the information bandwidth. In this way the speckle pattern, which originates from the interference and causes modal noise, will become "invisible" to the receiver. The modal noise can also be reduced by using broad linewidth VCSELs. This was taken into account when choosing proper light sources for the network realization. Integrated multimode optical devices based on polymers are used that have potential for low-cost realization [6], [7]. Moreover, fiber-to-the-chip coupling is facilitated using those devices since they contain larger waveguides commensurate with the multimode fiber cores.

This paper is organized as follows. Two architectures for FTTD application are introduced in Section II. Solutions for 
two principal problems, namely, synchronization and collision detection, are discussed in Section III. In Section IV, the functionality of both analog and digital parts of the physical layer device are described. Practical implementation and experimental results are presented in Section V. In Section VI, we review the results and give suggestions for a definitive implementation.

\section{Two FIBER-TO-THE-DESK ARCHITECTURES}

To facilitate a smooth transition from existing copper-based networks to future optical LANs, we have chosen Ethernet technology since it is the most successful networking technology to date and has been deployed in nearly every corporate and campus network [8]-[10]. The use of Ethernet technology provides low-cost high-performance architectures.

A carrier sense multiple access with collision detect (CSMA/CD), on which Ethernet is based, provides the equal-priority access to shared medium. The shared medium however, is not used in fast Ethernet switched networks where collisions occur only in switches. Introducing switches has the disadvantage that they require power supplies and regular maintenance, and a large number of switches can increase costs. Since copper-based cables cannot reach more than 100 $\mathrm{m}$, more switches have to be placed to connect larger distances. Another disadvantage of switched networks is delay in the switches. New switches now on the market overcome this problem, but some delay will always be present compared to passive star couplers, which in passive optical networks introduce almost no delay. These reasons have led us to base our new network architectures on low-cost passive integrated multimode optical couplers that require neither an electrical supply nor extra maintenance and can be easily coupled to multimode fibers. Passive star couplers also offer us the possibility for point-to-multipoint connection.

The first proposed optical LAN is the optically transparent network of Fig. 1.

Network segmentation is done by means of coarse wavelength-division multiplexing (WDM). The network configuration is star formed and segmented in ministars, which offers geographical advantages. Each segment of the LAN uses its own assigned wavelength. A common signal transfer path to the router is created by inserting a multimode coarse WDM (de)multiplexer. In this way cabling length, which is determined by the maximum allowable signal delay prescribed by the standard, is reduced. The stability requirements for the lasers are not so stringent since multimode fibers are used and the wavelengths are not so densely packed as in public switched telephone network applications.

The ministars consist of the end nodes that show an extensive mutual exchange of information. The geographical spread of these nodes is small, so that a long common transmission line to the router is allowed. The stars are implemented using passive optical star couplers. Electronic circuitry is only required in the router, which facilitates maintenance.

One disadvantage of this architecture is found in the fact that the different NICs in the end nodes require different wavelength light sources for each ministar. Another is the limited span of the

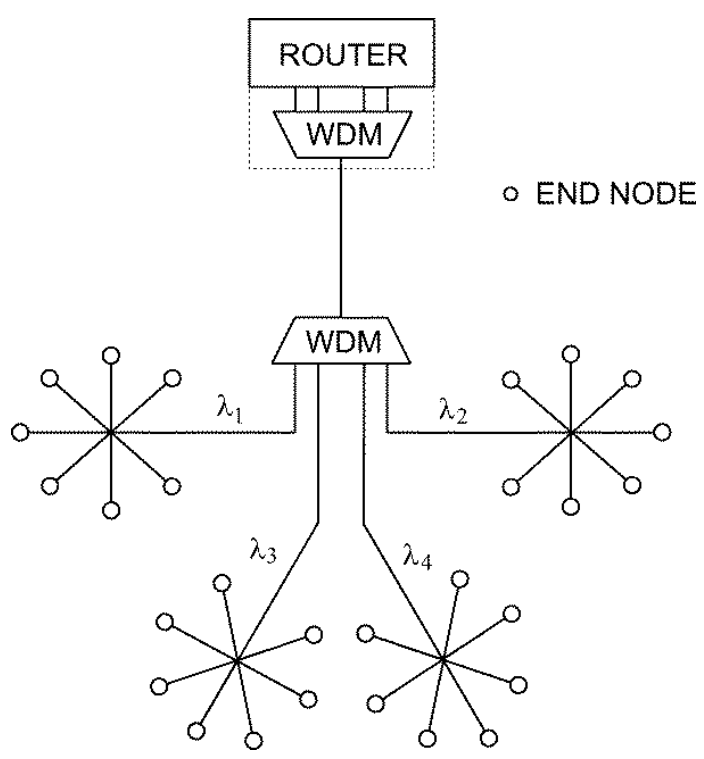

Fig. 1. Optically transparent LAN for FTTD application based on multiple single-wavelength optical stars using a different wavelength for each ministar.

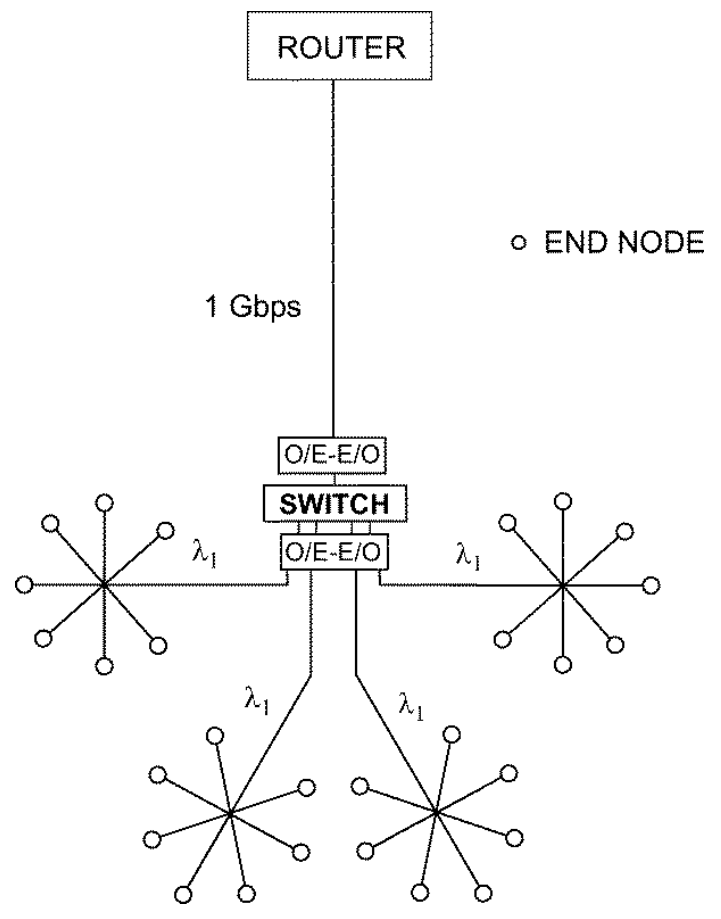

Fig. 2. Optical LAN for FTTD application based on single-wavelength star using the same wavelength for each ministar.

network because both the end nodes and the router belong to the same collision domain, limited in length by the standard.

To overcome these drawbacks, an alternative architecture is presented in Fig. 2.

In this case, an electronic switch is introduced with a gigabit Ethernet uplink on a single fiber pair to the router. The uplink can also be made using a single fiber at the cost of the power budget, since an extra splitter/coupler pair has to be introduced to split the up- and downstream traffic. The cost is a critical factor when choosing a single-fiber or a fiber-pair solution, although this specific cost will be shared among all the users in the LAN. 
The main advantage of this architecture is that the NICs can be identical for the end nodes all over the LAN since all the ministars use the same wavelength. The network span is larger since the end nodes in the ministars and the router do not belong to the same collision domain; thus the distance from the end nodes in the ministar to the switch can be up to $400 \mathrm{~m}$. The total bandwidth is shared only among the users within the same ministar. The number of users is limited by the losses in the star coupler and is typically about 16 to 32 users. The disadvantage is that electronic equipment, including the power supply, must be installed outside of the router cabinet, but there is only one switch that connects all the ministars, whereas the end nodes are interconnected by means of the passive star coupler.

Two other possibilities for overcoming the problems of the first architecture come to mind. One is the introduction of an electronic bridge instead of an electronic switch. This is a cheaper solution with much smaller throughput. Another is the introduction of a fast Ethernet switch that supports parallel switching with an extra coarse WDM (de)multiplexer between the switch and the router to support parallel transmission from and to different ministars.

The overall cost and specific application will guide users in choosing among the proposed architectures. In all the architectures the fiber lengths are limited by neither dispersion nor attenuation but mostly by the protocol itself, as can be seen in Fig. 3.

Even with 16 users per ministar, we can still have more than $1 \mathrm{~km}$ of fiber for the bit rate of $125 \mathrm{Mb} / \mathrm{s}$ prescribed by the standard [1].

The proposed architectures require changing the physical layer implementation in the ISO-OSI model while the medium access control (MAC) layer and higher layers remain unaltered. This facilitates network realization and lowers the overall cost. Such a realization is supported by the medium-independent interface (MII) connector between the MAC and the physical layer by means of which several different physical layer devices (PLDs) can work on the existing NIC. Although in the final realization cost consideration will lead to integration of the Physical and Data Link Layer, we have concentrated on the realization of a new PLD that can be attached to the existing NIC equipped with the MII interface. Two stumbling blocks, synchronization of bursty traffic and collision detection, had to be resolved.

\section{SynchronizATION AND COLLISION DeteCtion}

Since both architectures are based on passive star couplers, we have a common shared medium for the signal transmission, inherited from the original 10BASE-T Ethernet standard. This is in contrast to 100BASE-T Fast Ethernet, where each user has a separate path to a bridge or a switch. In the latter, synchronization is facilitated since continuous transmission between the end node and a bridge or a switch can be maintained. When no packets are transmitted, the IDLE signal is transmitted for synchronization. On the other hand, the use of a shared medium in our new architectures excludes this possibility and we had to find a solution for the synchronization of burst traffic, since the end node is allowed to transmit only when it has a packet.

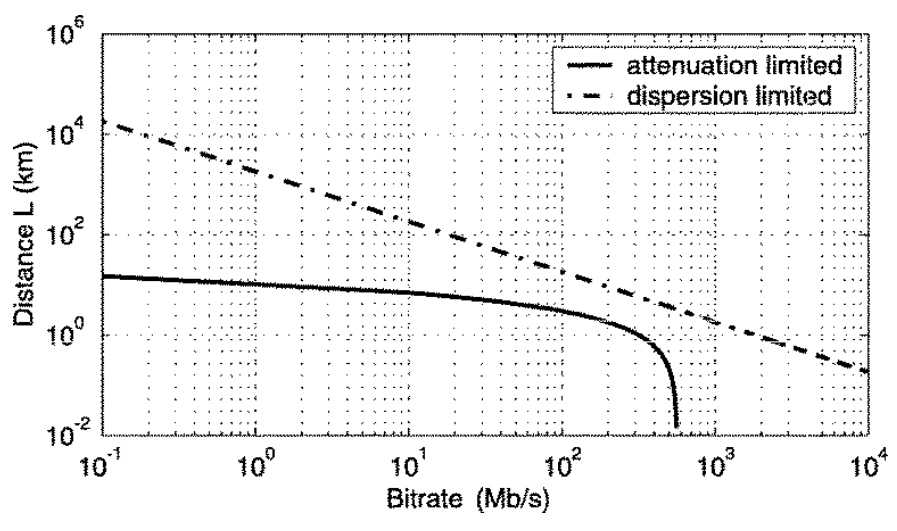

Fig. 3. Maximum fiber length versus bit rate for the system with 16 end nodes in the ministar. Both attenuation in the system and dispersion in the fiber are taken into account.

Another problem to be solved was collision detection. In the existing 100BASE-T IEEE media specification, collision detection has been performed in the digital part of the PLD and is based on detection of simultaneous transmission and reception of data signals. We could use a similar approach but the received data cannot be recovered at the beginning of the packet without being synchronized; that would have caused a large delay in the collision detection process, decreasing the network throughput.

\section{A. Synchronization}

The simplest solution of the synchronization problem is to use a multiplexing technique allowing simultaneous transmission of clock and data signals: for example, use WDM and introduce an extra wavelength for clock signal transmission, subcarrier multiplexing, or even spectrally encoded optical code-division multiple access [11]. Although these solutions might have been simple to realize, they would most certainly have led to an increase in overall network cost, something that we wished to avoid.

To keep the costs low, we propose using the embedded clock transport (ECT) technique, where an analog sinusoidal signal is simultaneously sent in addition to the data signal during the whole packet duration. Provided that the clock signal in the PLD at the transmitting side has been generated using this sinusoidal signal, we can recover data at the receiving side. Separation of the encoded data signal, sent in packets, and the synchronization signal can be performed by using simple filtering, given that the frequency content of the signals does not overlap. Introducing the extra synchronization signal improves detection at the cost of increased power consumption. The ECT technique is simple and effective and scales very well with bit rate, so this synchronization technique can also be used when upgrading the networks introduced here so that they can accommodate the gigabit Ethernet protocol.

The actual bit rate of the signal transmitted through the fiber when using the fast Ethernet protocol is $125 \mathrm{Mb} / \mathrm{s}$. To generate these data, a $125-\mathrm{MHz}$ clock is used. Since the data signal is NRZ-I encoded after 4B/5B coding [1], its power spectral density will have a quadratic sinc shape with the first spectral null appearing at $125 \mathrm{MHz}$. This offers us an opportunity to insert a synchronization signal at this frequency, as shown in Fig. 4. 


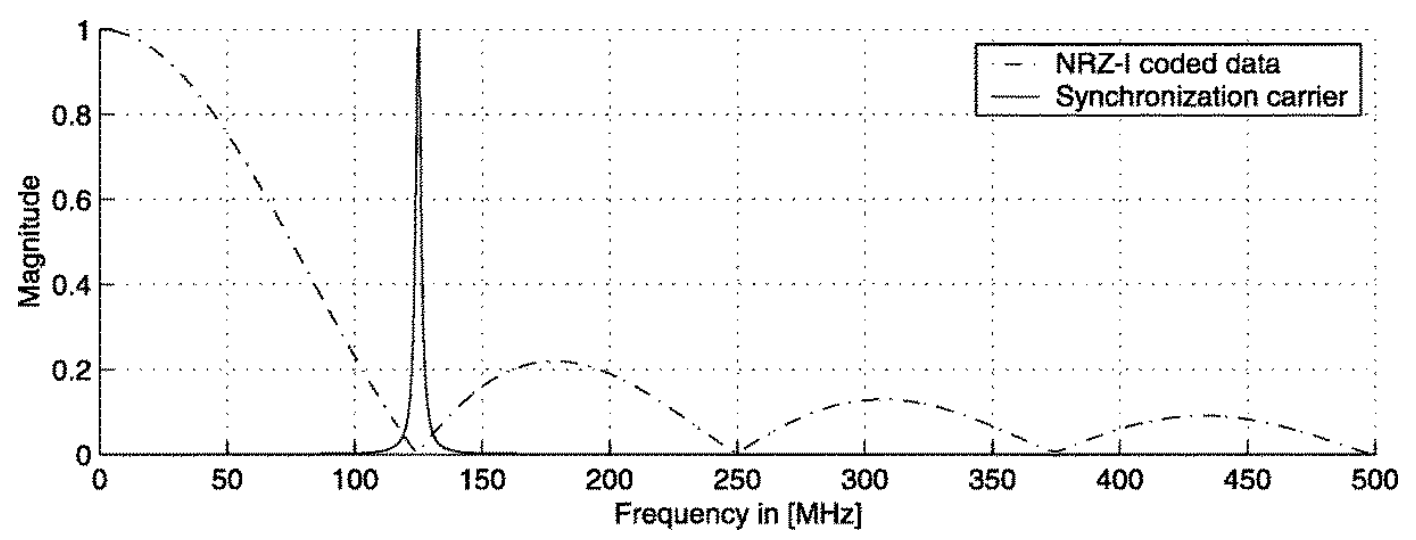

Fig. 4. Power spectral densities of the NRZ-I encoded signal and synchronization signal.

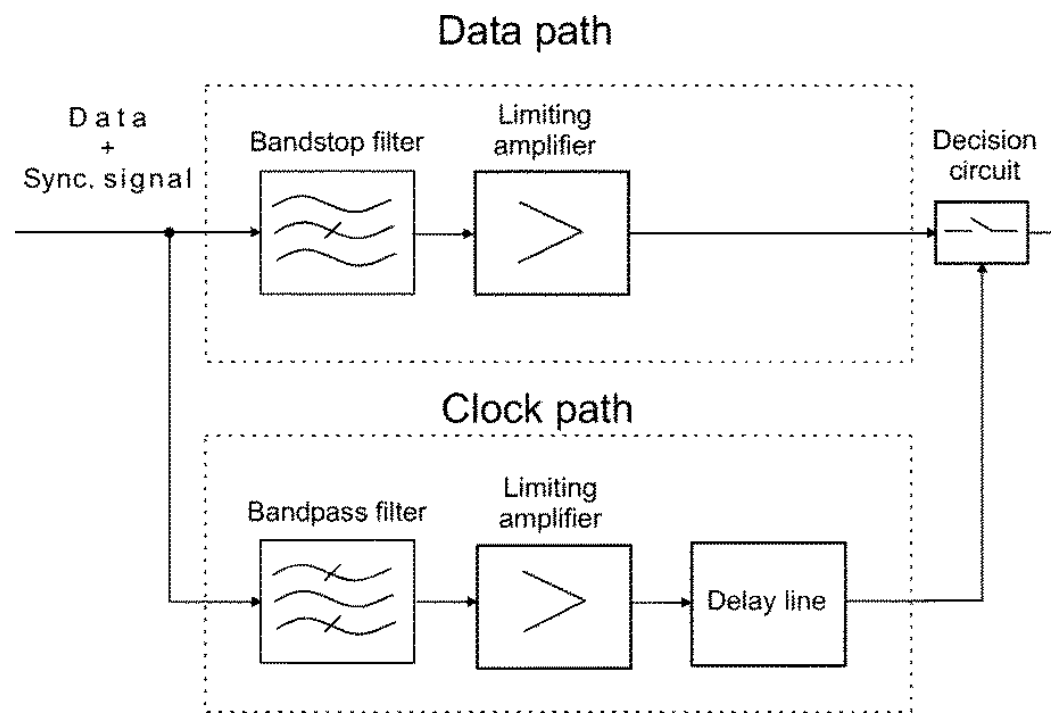

Fig. 5. Block scheme for the clock and data recovery circuit in the receiver.

As can be seen from Fig. 4, a narrow-band 125-MHz synchronization signal cannot seriously affect received data signals, keeping in mind that $95 \%$ of the data signal power is contained in the spectrum between the frequencies of 0 and 0.75 times the data rate. This means that we can easily separate the signals by using two filters: a bandpass filter with a center frequency of $125 \mathrm{MHz}$ for the synchronization signal and a bandstop filter with the same center frequency for the data signal. This concept is illustrated in Fig. 5.

The recovery time of the synchronization signal will depend on the type of bandpass filter used and its response time. The best choice can be found by studying the tradeoff between quality factor and filter response. On the one hand, we would like to have a filter with a rather narrow passband to eliminate the presence of the data signal; on the other hand, we need a quick response time of the filter to achieve fast synchronization. The amplifiers placed after the filters serve to make a clock signal out of the synchronization sinusoidal signal in the clock path and amplify the signal amplitude in the data path. The delays in both paths might be different, and this is the reason we have added an extra delay line to synchronize the data and the clock signals. Finally, a decision circuit is used for data recovery.

\section{B. Collision Detection}

Collision detection is a mechanism that has been added to CSMA/CD, which in turn has been implemented in the MAC layer. This mechanism ensures that a given station will not start sending packets as long as another station in the same collision domain is occupying the shared transmission medium. Furthermore, it will discontinue transmission in case an incoming signal from another station is detected at the same time.

Several collision detection methods have been proposed for fiber-optic CSMA/CD networks [12]. We chose to use a directional coupling method in combination with average power sensing due to the simplicity of implementation.

The directional coupling method uses a special coupling, or interconnection technique, which ensures that the transmitting station can listen to all stations but itself. Collision detection in this system is straightforward. If a station senses data at its receiver while it is transmitting, it means that some other station is also transmitting and thus a collision has occured [12]. But, at the beginning of the receiving process, we do not have synchronization; thus no data bits can be recovered. To detect a collision, we have to combine another method, average power sensing, in which detection of the incoming signal is determined based on the average power carried. 
To implement this type of collision detection, a special reflective-type star coupler has to be used. The idea for implementing such a coupler has been proposed in [13], but we want to realize it as a low-cost multimode integrated-optic component. Depending on the optical star design, one or two fibers can be used per user for signal transmission. In the case of a one-fiber system, fiber cost is lower but an additional $2 \times 2$ coupler must be placed before the transceiver in the NIC to split up- and downstream traffic.

Another condition for this type of collision detection is that all the sources in a given ministar use laser diodes of identical wavelength, which is exactly the case in our architectures. In order to predict a link budget that fits all the hosts, the output power of laser diodes in each of the hosts should be nearly identical. Fluctuations in the transmitting lasers will always occur and must be reflected in the threshold level for collision detection.

To implement the collision detection mechanism, we have to calculate optical power level at the photodetector in both of the architectures. It is obvious from Figs. 1 and 2 that optical signals in every ministar are physically separated from the signals in other ministars, so collisions will occur only within a single ministar. To calculate power levels, we have to take into account losses in the fiber, connectors, star coupler, and the first architecture WDM (de)multiplexer. The main signal attenuation will be in the passive star couplers [7], especially if more users are connected to the same ministar. Laser output power must be sufficiently large to compensate for the losses. In the first proposed architecture, lasers that are built in the router NICs must supply greater output power to compensate for the WDM losses such that the signal received from the router does not differ much in power level compared to the signals received from another end node within the same ministar. To include degradation of the components with aging, some margin must also be taken into account.

In an ideal case, when a signal is received that is larger than a certain noise level, we will sense the carrier from another end station. An appropriate signal RX_COL that goes to the MAC layer will be set high to indicate that an other end station is already occupying the transmission medium. In the case that no other end station is transmitting, we will have no signal on the line. On the other hand, if the level of the received signal when a packet is transmitted is high enough, carrier sensing will be simple. The digital part of the PLD now checks whether the NIC is transmitting and, if that is the case, activates the collision mechanism in the MAC layer. If the end station is not transmitting, it defers transmission according to the CSMA/CD protocol. But, sending the synchronization signal requires that the laser work in its linear regime, which means that there will always be some light transmitted, even when no data packet is sent. Although this power level is low, it has to be taken into account when more users are connected to the same ministar. To sense the carrier, we must have signal-level differences in the case that no packet is transmitted and in the case that the signal is transmitted. Taking into account all the variations in signal levels, caused by the lasers and passive optical components we have to place a threshold between the two levels such that carrier sense signal is high when the threshold is crossed. This is

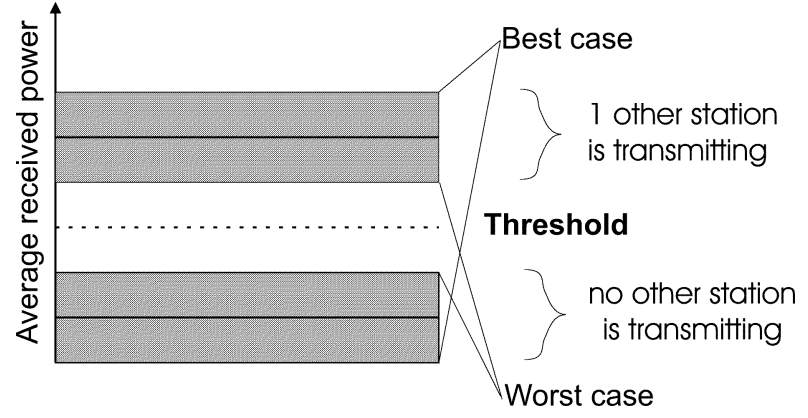

Fig. 6. Threshold-level determination for the carrier sense.

presented in Fig. 6. The threshold level will mainly depend on the type of lasers used in the system and the number of users in the ministar.

Since the data packet is accompanied by the synchronization signal, there are three possibilities for measuring the received signal level that will be used to determine the threshold. We can either separately measure the average value of the data and the synchronization signal after filtering or the level of the combination of both before that.

Provided that the signal is encoded and possibly scrambled, it is very difficult to measure the mean of the data signal, for example, after integration, since the value varies depending on the integration constant. The larger the integration period, the more accurate the result is, but the time for the carrier sense is also longer and that will cause a decrease in throughput. On the other hand, if we want to measure the mean value of the synchronization signal in the absence of the data signal, since it should yield a more constant value after a shorter integration time, we will get zero since the dc component is eliminated by means of a bandpass filter. Some methods can be used to overcome this problem, but this would only complicate the design. The simplest method for determining the received signal level is to measure it before the signals are split into data and synchronization components (Fig. 5). Tapping a part of the signal in the receiver and bringing it to the integrator is the best way to determine the received signal level. Although the value obtained varies with the integration constant due to changes in the data, we can set the threshold based on the level obtained from the synchronization signal only and the mean data value will only contribute to exceed that threshold in the case that the data signal is unipolar. If the threshold is reached, then the carrier is sensed. Based on this signal, we can detect a collision by sensing the simultaneous presence of the transmitted signal.

\section{ANALOG AND Digital PARTS OF THE PLD}

\section{A. Analog Part of the PLD}

Introducing an extra synchronization signal requires modification of the analog part of the transmitter in PLD, with respect to the original 100BASE-T IEEE media specification, since here the combination of the data and the sinusoidal signal has to be transmitted. Sending a sine-shaped signal requires that the laser operating point always lays in the linear part of the P-I curve, which is presented in Fig. 7. 


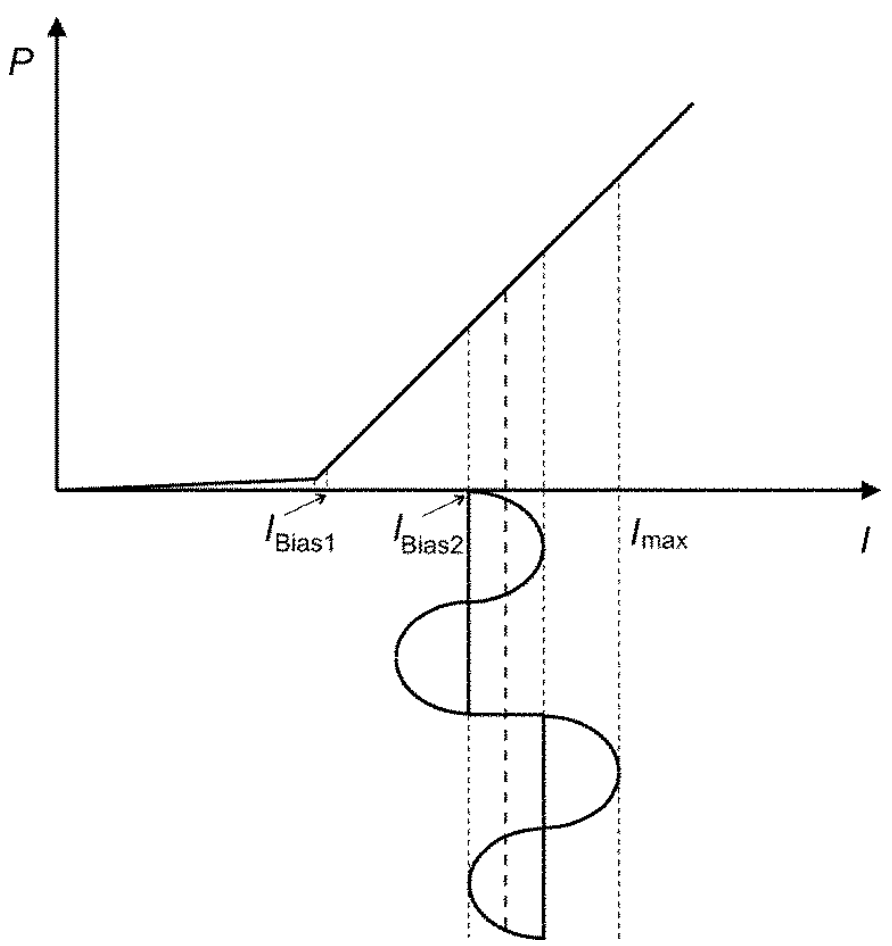

Fig. 7. Direct modulation of the laser with combined data and synchronization signals.

To simplify the implementation, the laser is usually directly modulated using on-off keying modulation format with various modulation depths. In order to facilitate collision detection, the difference in transmitted power when data packet is transmitted or is not must be as large as possible. If the bias current of the laser is chosen such that the operating point is in the middle of the linear part of the $\mathrm{P}-\mathrm{I}$ curve, we will have light even when there is no data packet to be transmitted and the difference between the signal levels with and without data would be very small. This would make it very difficult to set up a threshold level for carrier sensing. The threshold level must be kept as low as possible when the data are not transmitted in order to facilitate carrier sensing and eventually collision detection.

A solution to this problem has been found in using two bias currents. One will set up the operating point when there is no data to be transmitted ( $\left.I_{\text {bias1 } 1}\right)$, and the other $\left(I_{\text {bias2 }}\right)$ when data are transmitted, as shown in Fig. 7.

The first bias current will be slightly larger than the threshold current. Emitted power will be very low, and the laser can respond to quick changes in operating point when necessary, since it has already been turned on. Due to this small emitted power, we will receive the summed powers from all the other end stations in the ministar, even when the end stations are not transmitting data. If the power level is much smaller than the power received from an end station transmitting a data packet, added to the power from the other end stations that are not transmitting data, we can easily set up the threshold level to sense the carrier. When the end station is ready to transmit the packet, the bias current has to be changed such that the sinusoidal synchronization signal can be sent without distortion. The condition to be met is that there is no clipping even in the presence of the data signal. This means that total signal amplitude must remain

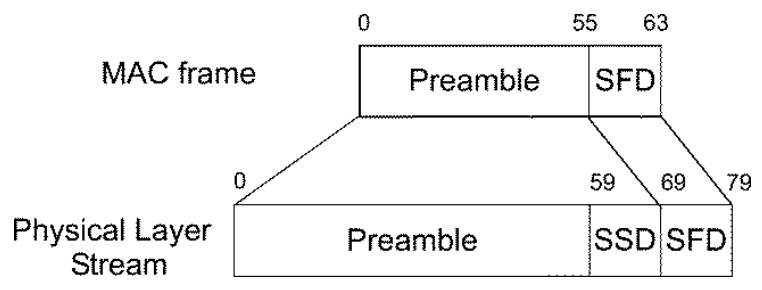

Fig. 8. Start of stream delimiter is inserted just in front of the start of frame delimiter to be sure that synchronization has been achieved.

within the linear part of the P-I curve. This leads to a tradeoff between the signal amplitude and the bias current. In order to facilitate detection, it is better to have a larger difference between the logical levels of the data signal. On the other hand, since we use only the sum of the zero mean value synchronization signal and the bias current for carrier sensing, a larger bias current would facilitate collision detection.

The main analog parts of the receiver-the clock, data recovery, and carrier sense circuit-have been presented in Section III.

\section{B. Digital Part of the PLD}

The digital part of the PLD has to accommodate both signals coming from the MAC Layer via MII for the transmitter and process signals coming from the analog part of the receiver before proceeding to the MAC layer. All the signals passing through the MII interface must be in conformance with the IEEE $803.2 \mu$ standard. The interface accommodates two 4-bit data buses and several control signals to provide the communication needed between the MAC and Physical layer [1]. There are several differences between the standard PLD and our new PLD.

First, we do not use an IDLE signal for synchronization in order to avoid constant collisions. Moreover, we do not require a JAM signal, which is used by the hub to indicate that some other station is transmitting, thereby commencing the collision detection process. This is due to the fact that we have here a point-to-multipoint connection and all the end stations sense the shared medium at the same time. Another difference is that we do not require extra data encoding, such as MLT-3, to reduce bandwidth, since we have enough bandwidth available in the optical fiber. Data scrambling can also be avoided. The preamble of the data packet is slightly changed due to the synchronization problem. Since the synchronization will not be established during the first several bytes of the data packet, we cannot place a start of stream delimiter (SSD) at the beginning of the packet, as it will not be detected. In the original standard, this has been used to distinguish data packets from the IDLE signal. To detect the beginning of the packet, the safest way is to put SSD just in front of the start of frame delimiter (SFD), so we can be sure that the synchronization has been achieved at that point and that the packet can be recognized. This is shown in Fig. 8 .

Another extra feature that we have inherited from the original 10BASE-T Ethernet is jabber detection. This is important for disconnecting end stations that are continuously transmitting, leading to a constant collision. After some predefined period, Unjab time, a station can be connected again. To disconnect a station, a special signal is used that switches off the laser by decreasing its bias current. All the other functions stay the same. 


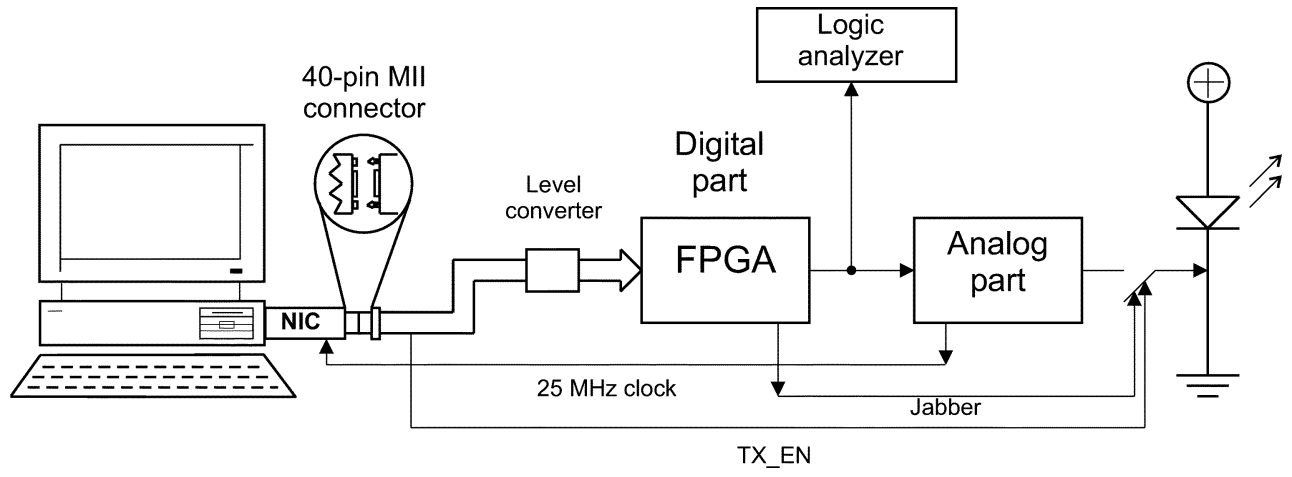

Fig. 9. Experimental setup for the transmission part of the new PLD.

We have to stress that PLDs must have an internal clock running at $125 \mathrm{MHz}$ that can be switched from free-running value to the value determined by the incoming synchronization signal, which is specified in the standard. This is important since we have a synchronization signal only during the duration of the data packet.

\section{REALIZATION, EXPERIMENTS, AND DISCUSSION}

Experiments were separately conducted for the transmitter and the receiver in the PLD, in order to have a better insight in their functionality. Both the transmitter and the receiver consist of analog and digital circuitry. The digital part is implemented using a field-programmable gate array (FPGA) chip form the Altera APEX $20 \mathrm{~K}$ chip family and we have fabricated separate printed circuit boards (PCBs) for the transmitter and the receiver analog parts. A receiver comprises separate clock and data recovery circuits and the carrier sense circuit.

The experimental setup for the transmission part of the PLD is shown in Fig. 9. The NIC used in the experiment is equipped with the MII interface.

The digital data that are to be transmitted through the network in the form of fast Ethernet packet are applied via a 40-pin MII connector to the input of the PCB with the FPGA chip. The cable length between the MII connector and this PCB must be short enough to avoid extra delays in transmission. A logical level converter is placed in between to convert the transistor-transistor logic signals from the MAC layer to low-voltage differential signals that are brought to the FPGA input.

For proper functioning of the NIC, the analog part of the PLD provides a $25-\mathrm{MHz}$ clock, since the data coming from the MAC layer are clocked with this frequency. The actual data rate on the line is $125 \mathrm{Mb} / \mathrm{s}$, which means that the FPGA must also run at this clock frequency.

After SSD is added to the MAC frame, data are 4B/5B encoded, scrambled (can be switched off), and converted to a serial form with the data rate of $125 \mathrm{Mb} / \mathrm{s}$. The data stream proceeds toward the analog PCB circuitry for conversion to an optical signal. This data are monitored using a logic analyzer.

In the analog PCB, a synchronization signal is added to the data signal. After both signals are amplified, a dc current $\left(I_{\mathrm{bias} 2}-I_{\mathrm{bias} 1}\right)$ is added for appropriate biasing. Such a signal is added to the biasing current $I_{\text {bias1 }}$ when data packets are to be transmitted. This has been done via a switch that is controlled by the TX_EN signal from the MAC Layer. When a packet is to be transmitted, a TX_EN signal is high and that opens the gate of a junction field-effect transistor that supplies three extra currents to the anode of the laser.

When there is no packet to be transmitted, only the small bias current $\left(I_{\text {bias1 } 1}\right)$ is flowing through the laser, contributing only to increase of the dc level of the received signal.

Another signal that controls the switch is the Jabber signal, which is activated to open the switch when the end station, due to malfunction of the NIC, is transmitting a data stream much longer than the longest packet prescribed by the standard.

A laser that is placed at the front-end of the transmitter is directly modulated by signals from the analog PCB. Two types of short wavelength lasers working at 790 and $850 \mathrm{~nm}$ have been used. Two different operating wavelengths were necessary for realization of the first architecture shown in Fig. 1. Choosing the wavelengths far apart from each other facilitates the realization of the multimode coarse WDM (de)multiplexer. The laser operating at $790 \mathrm{~nm}$ is of the Fabry-Pérot type, while the laser operating at $850 \mathrm{~nm}$ is a VCSEL. The two lasers have different values of bias current, which has to be taken into account when setting the operating points of the lasers. Nevertheless, they both have very linear P-I curves. The spectrum of both lasers is broad enough to avoid speckle noise.

We also measured the relaxation oscillation frequency of the Fabry-Pérot laser to determine whether it is far beyond the data spectrum. The results showed that it is at least $1.6 \mathrm{GHz}$ for the smallest bias current, which means that the modal noise may be negated. The spectral linewidth of the laser is about $4 \mathrm{~nm}$, while the linewidth of the light from the VCSEL is $3.5 \mathrm{~nm}$.

To analyze the effect of modal noise, we have placed a camera at the fiber end and observed the intensity of the light in the cross-section of the multimode fiber. Almost no speckle pattern was visible, which makes the system less vulnerable for fiber misalignment and dust particles.

We used in the experiment a 50/125 $\mu \mathrm{m}$ silica graded-index multimode optical fiber with attenuation coefficient of $2.5 \mathrm{~dB} / \mathrm{km}$.

The experimental setup for the receiver is shown in Fig. 10. In the same figure, we have presented more details of the analog part of the receiver.

A photodetector with typical responsivity of $R_{\mathrm{pd}}=$ $0.4 \mathrm{~mA} / \mathrm{mW}$ is pleased at the front-end of the receiver. In the analog part of the receiver, carrier sense and clock and 


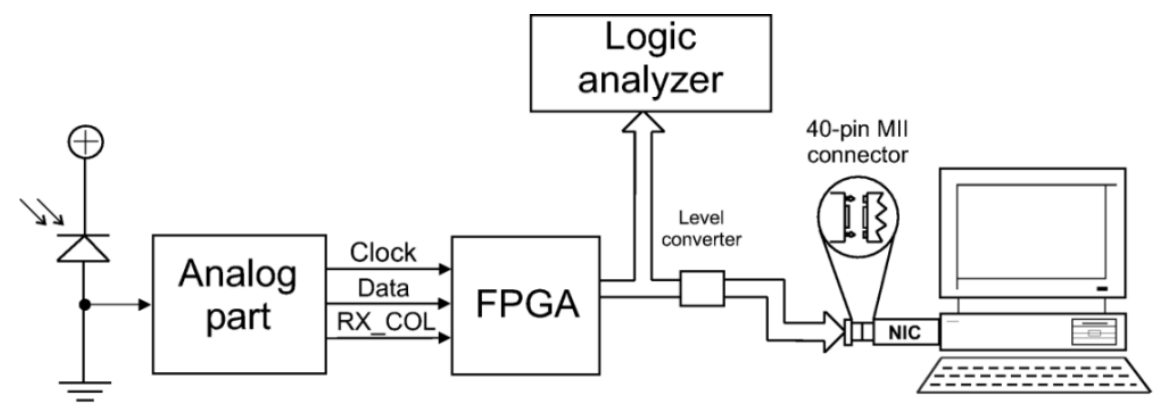

(a)

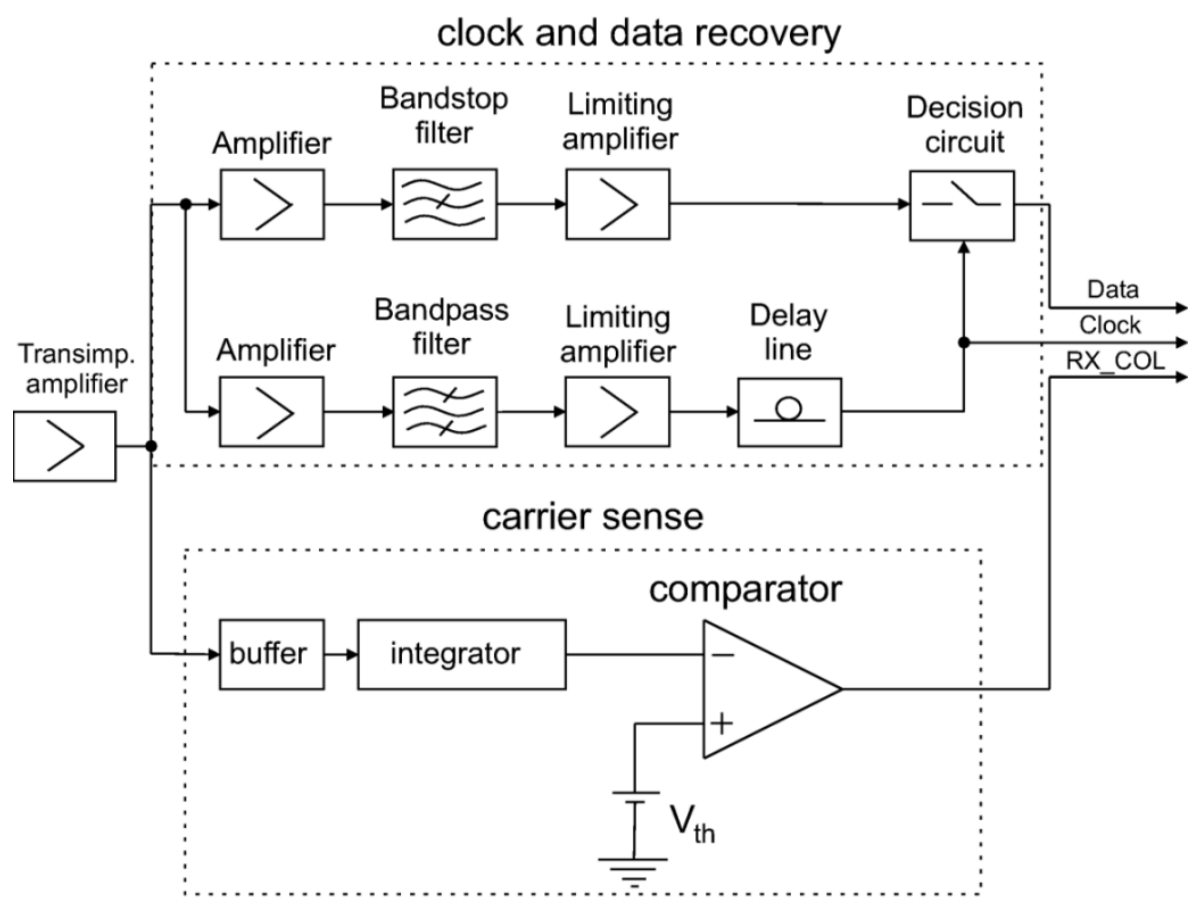

(b)

Fig. 10. (a) Experimental setup for the receiving part of a new PLD in FTTD network. (b) Analog part of the receiver.

TABLE I

OpticAl Power Received AT THE PLD IN ONE OF THE END NODES IN THE Ministar When I) No Other END Station Is SENDing Data, II) ONE OUT of EIght End Stations Is SENDing Data, III) THE Router Is SENDING DATA Signal (ONLY VALID FOR THE FIRST ARChITECTURE)

\begin{tabular}{l|c|c|c}
\hline & I & II & III \\
\cline { 2 - 4 } VCSEL/LD power & $15.9 \mathrm{dBm}$ & $0.1 \mathrm{dBm}$ & $5.0 \mathrm{dBm}$ \\
\hline star coupler loss $(8$ users $)$ & $10.5 \mathrm{~dB}$ & $10.5 \mathrm{~dB}$ & $10.5 \mathrm{~dB}$ \\
fiber loss & $1.0 \mathrm{~dB}$ & $1.0 \mathrm{~dB}$ & $1.0 \mathrm{~dB}$ \\
connector pairs $(4 \times 0.5) \mathrm{dB}$ & $2.0 \mathrm{~dB}$ & $2.0 \mathrm{~dB}$ & $2.0 \mathrm{~dB}$ \\
WDM losses & $0.0 \mathrm{~dB}$ & $0.0 \mathrm{~dB}$ & $4.9 \mathrm{~dB}$ \\
\hline power at the receiver & $-29.4 \mathrm{dBm}$ & $-13.4 \mathrm{dBm}$ & $-13.4 \mathrm{dBm}$ \\
\hline
\end{tabular}

data recovery are implemented. Signals obtained are further transported to the digital part, where the signals are processed, and via the MII connector sent to the NIC. The packet content is monitored using a logic analyzer.

After passing through the transimpedance amplifier, the received signal is split in three. One part goes to the carrier sense circuit and a second part goes to the clock and data recovery circuit, where it is further split in a data and clock recovery path.

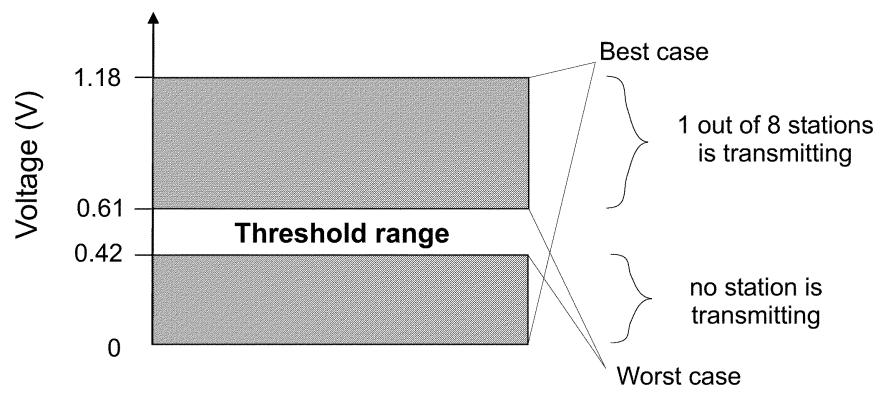

Fig. 11. The range of threshold levels in the analog part of the PLD in the end nodes that are directly connected to the multimode star coupler.

The carrier sense circuit contains amplifiers and an integrator that consists of two cascades of RC LP filters. The signal obtained is applied to the comparator input, where its level is compared to the predefined dc value representing the threshold obtained previously in Fig. 6 .

In the receiver, power levels at the input of the photodetector have to be calculated based on measurements. To simplify the 


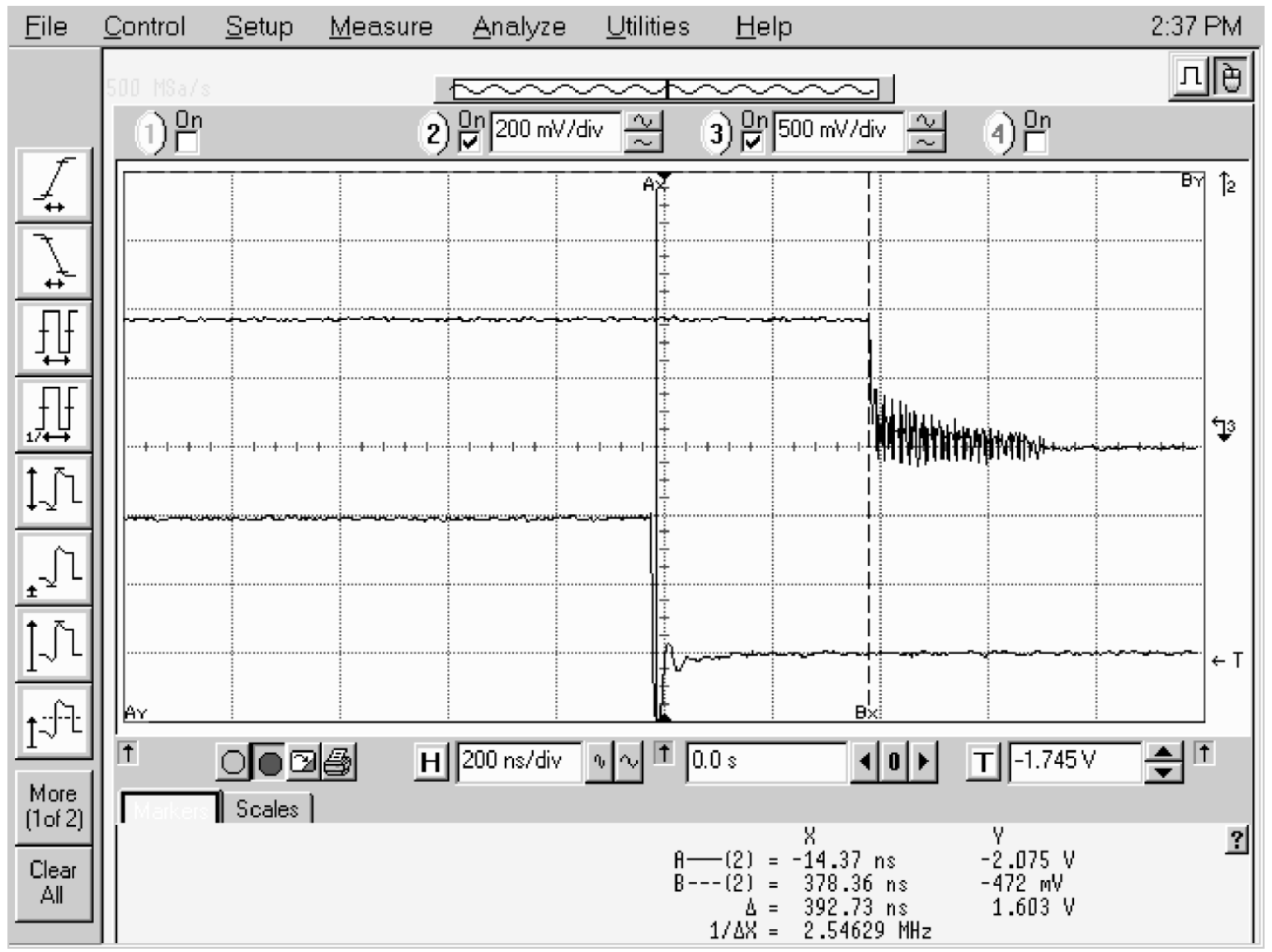

Fig. 12. Delay between the TX_EN signal from the transmitter in one of the end stations in the ministar and the CRS signal at the receiver in another belonging to the same ministar.

TABLE II

Bit Delay Constraints: MDI to MII Delay ConstrainTs (EXPosed MII, HALF-DUPLEX MODE)

\begin{tabular}{c|c|c}
\hline $\begin{array}{c}\text { Sublayer } \\
\text { measurement } \\
\text { points }\end{array}$ & Event & $\begin{array}{c}\text { Max } \\
\text { (bits) }\end{array}$ \\
\hline \hline MII $\Leftrightarrow$ MDI & TX_EN sampled to MDI output & 8 \\
\hline & MDI input to CRS assert & 17 \\
\hline & MDI input to CRS de-assert (aligned) & 22 \\
\hline & MDI input to CRS de-asserted (unaligned) & 17 \\
\hline & MDI input to COL assert & 17 \\
\hline & MDI input to COL de-assert (aligned) & 22 \\
\hline & MDI input to COL de-asserted (unaligned) & 17 \\
\hline
\end{tabular}

results, we have calculated the power when only the biased synchronization signal is transmitted since we can quickly determine the mean value of the signal after integration over several signal periods. This is presented in Table I. Measured values can be used to calculate the signal levels in the worst case situation when only the synchronization signal is transmitted through the ministar. Digital data that are added to the synchronization signal increase the mean value of the received signal that should exceed the threshold when a packet is transmitted through the ministar. When the carrier is sensed, a signal RX_COL is set high.

The values presented in columns I and II of Table I apply to both architectures, while column III applies only to the first architecture since the end nodes and the router do not belong to the same collision domain in the second architecture.

The measured losses of the WDM (de)multiplexer include connector losses. Results show that we have typically $16 \mathrm{~dB}$ greater received power in the case where the packet is transmitted on the line, even when the digital data are not included. This gives us sufficient room to place a threshold. The threshold range can be calculated using the responsivity of the photodetector, amplification of the transimpedance amplifier, and integration constant of the integrator. The range of threshold levels obtained through measurements at the comparator input is presented in Fig. 11.

The absolute received power at the photodetectors for different end nodes actually varies. Results for the received power represent the worst case and are valid for receivers that are directly connected to the star coupler. To calculate the power at the receiver, in the router extra loss in the WDM (de)multiplexer has to be added.

To compensate WDM losses, a laser placed in the router must supply greater power than the lasers in the end nodes of the ministar, so that the threshold range will stay unchanged.

To prove that the carrier sense complies with the standard specifications, we have measured the delay between the TX_EN signal at the receiver and RX_COL signal at the transmitter. This is shown in Fig. 12. 


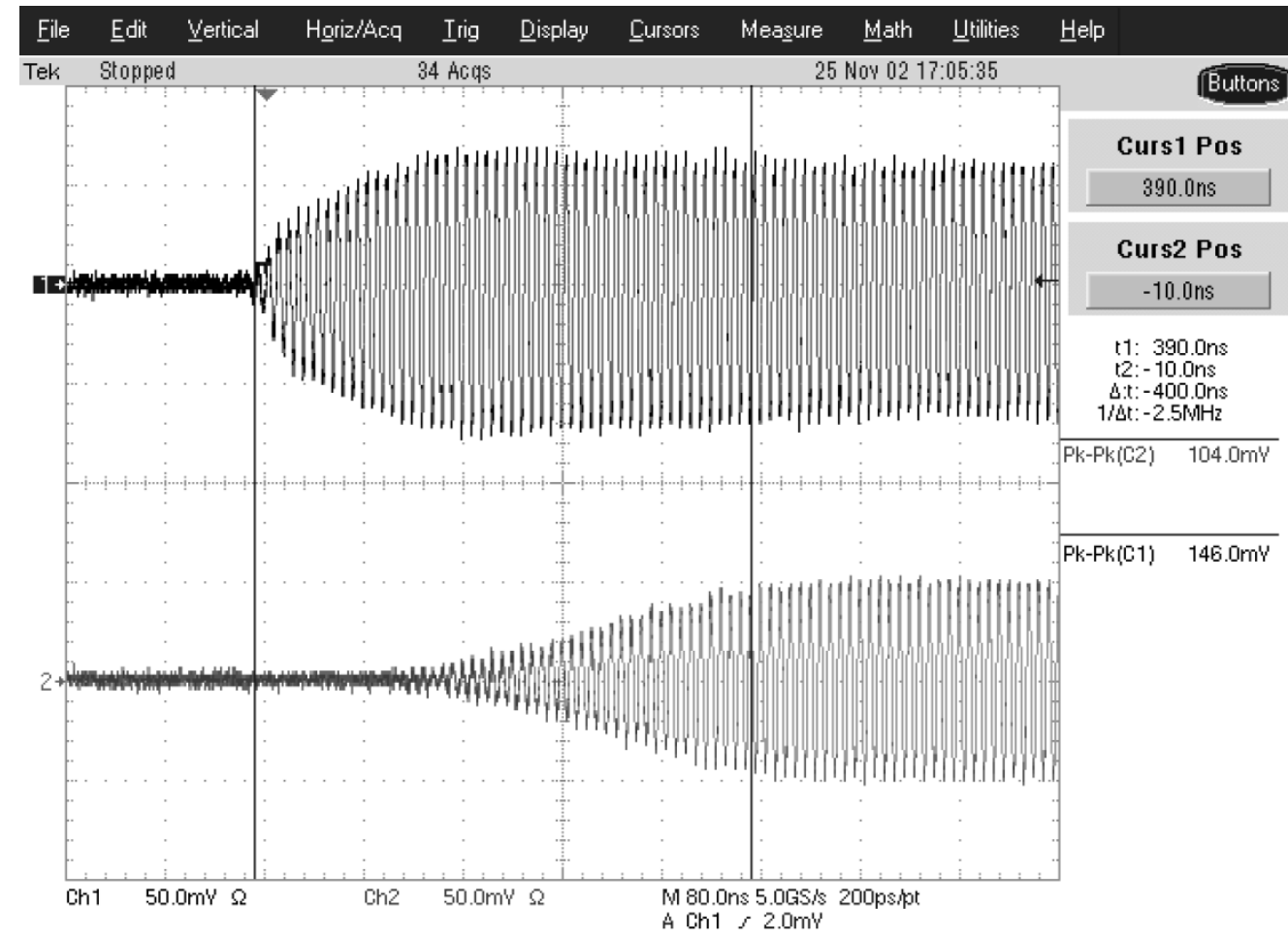

(a)

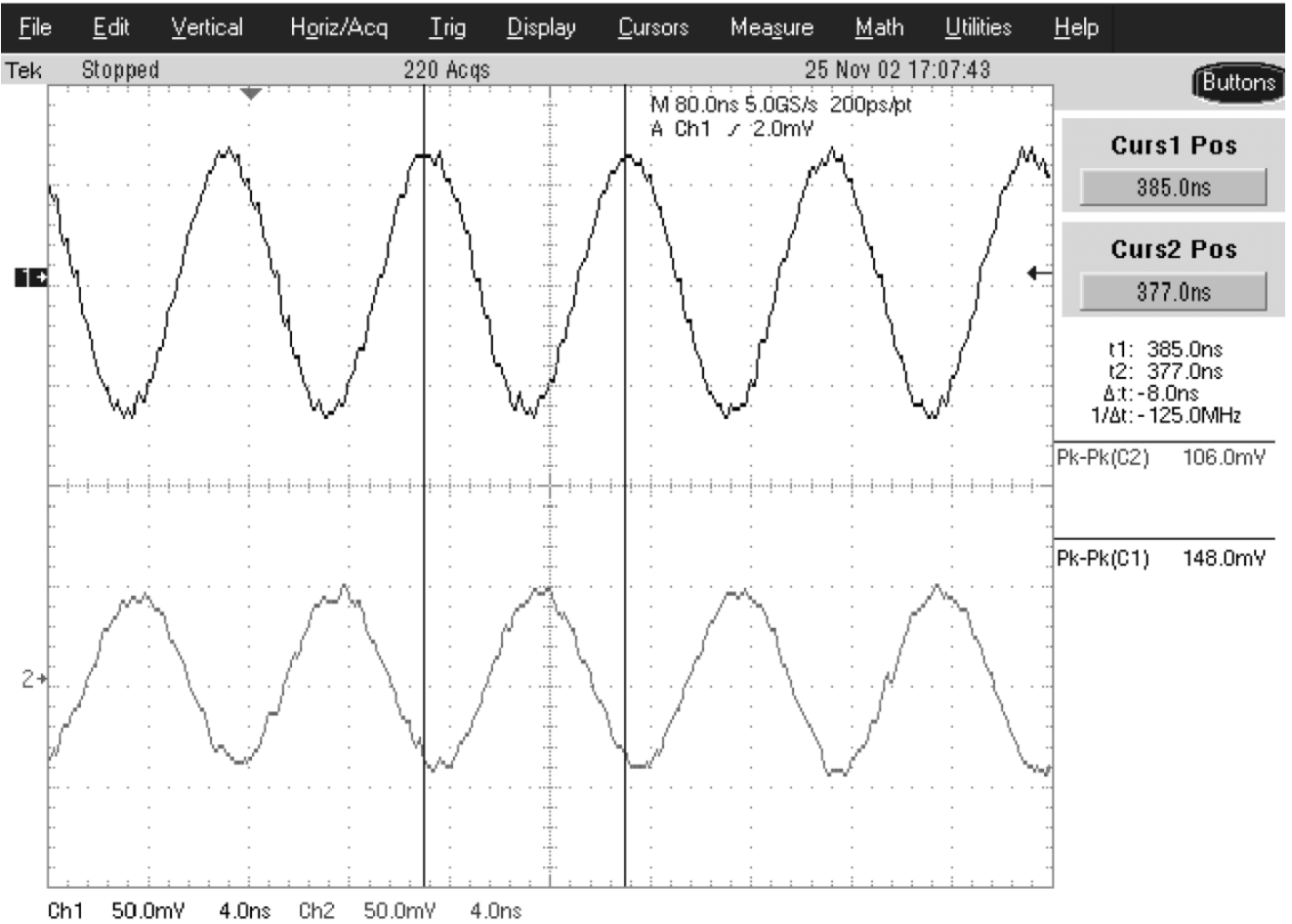

(b)

Fig. 13. (a) The response (lower trace) of the bandpass filter on the synchronization signal (upper trace). (b) Zoomed-in version of the signals at the moment the synchronization is achieved. 
It can be seen that the delay between the two signals is less than $400 \mathrm{~ns}$. This corresponds to 40 bits, since we have to keep in mind that $4 \mathrm{~B} / 5 \mathrm{~B}$ encoding is performed in the transmitter. Since we do not have a switch within a single collision domain and we do not require a JAM signal, this delay is very small. As far as the digital circuitry is concerned, all the delays comply with the standard [1], and are given in Table II.

The two parts of the incoming signal used for clock and data recovery are following amplification filtered by means of bandpass and bandstop filters with center frequency of $125 \mathrm{MHz}$, in the clock path and the data path, respectively. The bandpass filter used for clock recovery is a surface acoustic wave (SAW) filter with a 3-dB bandwidth of about $3 \mathrm{MHz}$, corresponding to the measured response time of about 400 ns. This is very important for clock extraction since we have to achieve synchronization within the first $480 \mathrm{~ns}$ of the data packet. The measured response of the filter on the input synchronization signal is presented in Fig. 13.

The amplified 125-MHz clock signal is then brought to a decision circuit for data extraction.

Part of the signal goes through the data path and is filtered by a passive fourth order bandstop filter [14] and amplified. The clock signal is properly delayed to ensure that both clock and data signal arrive at the decision circuit at the same time. The clock and the extracted data signal, together with the carrier sense signal, now go to the input of the FPGA chip to be processed for transmission to the MAC layer. A part of the FPGA output signal is monitored at the logic analyzer.

In the final network realization, for application in home and office premises, all the analog and digital parts of the PLD must be integrated, which will drastically decrease the size of the card and reduce the costs.

\section{DISCUSSION AND CONCLUSION}

We have presented fiber-optic LAN architectures for FTTD application suited for lowering the cost of bringing optical fiber all the way to the user. To achieve this, we have based our designs primarily on the most used Ethernet protocol. Moreover, we have used multimode fibers together with multimode polymer-based integrated star couplers and coarse WDM (de)multiplexers and short wavelength lasers and detectors. Using short wavelengths offers the possibility for integrating $\mathrm{Si}$ photodiodes with the receiver front-end using straightforward CMOS technology, something that will contribute to decreased overall implementation cost of the networks. Further decrease in costs is achieved through the low downtime of the network, low maintenance cost, and the possibility for future upgrade since optical fibers offer large bandwidth.

The architectures presented can also accommodate the Gigabit Ethernet protocol. To achieve this only NICs at the user's site and NICs in the router have to be replaced. It would appear that solutions for synchronization of bursty traffic and collision detection can be scaled to the gigabit Ethernet signaling rate of $1.25 \mathrm{~Gb} / \mathrm{s}$ facilitating a new gigabit Ethernet NIC realization.
Depending on demands, the user can choose among the architectures presented. The passive optically transparent architecture that we have presented first is cheaper to implement and is specially suitable for applications where no extra power can be supplied outside of the router cabinet and where maintenance costs are high. The second architecture offers better performance in a larger LAN, for example, in a bigger campus environment.

\section{REFERENCES}

[1] Carrier Sense Multiple Access with Collision Detection (CSMA/CD) Access Method and Physical Layer Specification, IEEE Standard 802.3, Sept. 1998.

[2] Optical Fiber Cabling Components Standard, ANSI/TIA/EIA-568B.3-2000 [Online]. Available: http://www.tiaonline.org/ standards/

[3] A. Savvas, "Fiber networks: Coming to a premises near you," Lightwave Eur., pp. 27-28, Oct. 2002

[4] J. Genoe, D. Coppée, J. H. Stiens, R. A. Vounckx, and M. Kuijk, "Calculation of the current response of the spatially modulated light CMOS detector," IEEE Trans. Electron Devices, vol. 48, no. 9, pp. 1892-1902, Sep. 2001.

[5] S. Radovanović, A. J. Annema, and B. Nauta, "3 Gbps monolithically integrated photodiode and preamplifier in a fully standard CMOS," in Proc. Int. Solid-State Circuit Conf. (ISSCC 2004), San Francisco, CA, Feb. 2004, to be published.

[6] S. Musa, A. Borreman, G. Pandraud, P. Knijn, G. Sengo, M. B. J. Diemeer, and A. Driessen, "Multimode fiber matched arrayed waveguides grating-based (de-)multiplexer for short distance communications," in Eur. Conf. Optical Communication, Copenhagen, Denmark, Sept. 2002, PLC 6.2.1.

[7] S. Musa, N. S. Lagali, B. Docter, G. Sengo, G. J. M. Krijnen, and A. Driessen, "Design and fabrication of low-cost, ultra-compact polymer based multimode $4 \times 4$ star couplers," in 10th Eur. Conf. Integrated Optics (ECIO), Paderborn, Germany, 2001, pp. 248-251.

[8] C. E. Spurgeon, Ethernet, The Definitive Guide. Sebastopol, Canada: O'Reilly, 2000.

[9] L. B. Quinn and R. G. Russsell, Fast Ethernet. New York: Wiley, 1997.

[10] G. Held, Ethernet Networks. New York: Wiley, 1998.

[11] I. Radovanović, G. Heideman, H. Siasi, A. Meijerink, and W. van Etten, "Addressable spectrally encoded optical CDMA system for application in access and local area networks," in Proc. URSI XXVII General Assembly, Maastricht, The Netherlands, Aug. 2002, D7.O.4., pp. 1047-1051.

[12] J. W. Reedy and J. R. Jones, "Methods of collision detection in fiber optic CSMA/CD networks," IEEE J. Select. Areas Commun., vol. SC-3, pp. 890-896, Nov. 1985.

[13] T. Hermes, "New reflective type star coupler for full duplex channels," Electron. Lett., vol. 27, pp. 2136-2138, Nov. 1991.

[14] A. I. Zverev, Handbook of Filter Synthesis. New York: Wiley, 1967.

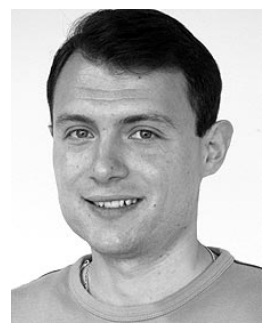

Igor Radovanović (S'99) was born in Peć, Serbia, in 1974. He received the B.Sc. and M.Sc. degrees in electrical engineering from the Faculty of Electronic Engineering, University of Niš, Niš, Serbia, in 1997 and 2000, respectively. He is currently pursuing the Ph.D. degree at the University of Twente, The Netherlands.

In 1999, he joined Telecommunication Engineering group, University of Twente, where he is engaged in research and development of new passive optical local-area networks for fiber-to-the-desk application. His research interests include optical code-division multiple accessing, Ethernet passive optical networks, broadband access network architectures, protocols, and signal and image processing. 


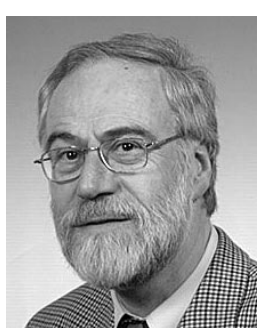

Wim van Etten (M'80-SM'91) was born in Zevenbergen, The Netherlands, in 1942. He received the M.Sc. and Ph.D. degrees in electrical engineering from Eindhoven University of Technology, Eindhoven, The Netherlands, in 1969 and 1976, respectively.

From 1969 to 1970, he was with Philips Electronics, developing circuits for oscilloscopes. In 1970, he became an Assistant Professor at the Faculty of Electrical Engineering Eindhoven University of Technology. From 1970 to 1976, he was engaged in research on the transmission of digital signals via coaxial and multiwire cables. Since 1976, he has been involved with research and education on optical fiber communications. In 1985, he has been an Associate Professor at the Eindhoven University of Technology. In 1994, he became a full Professor of telecommunications at the University of Twente, Enschede, the Netherlands. His current interests comprise optical communications, mobile communications, detection, and simulation of communication systems. He is the author or coauthor of more than 60 papers in international journals, conferences, and symposia. He was a member of organizing committees and program committees and Proceedings Editor of various conferences and symposia. He is first author of Fundamentals of Optical Fiber Communications (Englewood Cliffs, NJ: Prentice-Hall, 1991).

Prof. van Etten is a Member of the Dutch Institute of Electronic and Radio Engineers (NERG). He is President of NERG and Vice-Chairman of the Executive Committee of the IEEE Benelux joint chapter on Communications and Vehicular Technology. Two of his papers were selected for a collection of $57 \mathrm{key}$ papers on communications that appeared in the last 50 years in IEEE ComSoc publications.
Hennie Freriks, photograph and biography not available at the time of publication. 